\title{
Multiple reference genomes and transcriptomes for Arabidopsis thaliana
}

Xiangchao Gan ${ }^{1 *}$, Oliver Stegle ${ }^{2 *}, J_{\text {Jonas Behr }}^{3 *}$, Joshua G. Steffen ${ }^{4}$, Philipp Drewe ${ }^{3 *}$, Katie L. Hildebrand ${ }^{5}$, Rune Lyngsoe ${ }^{6}$, Sebastian J. Schultheiss ${ }^{3}$, Edward J. Osborne ${ }^{4}$, Vipin T. Sreedharan ${ }^{3}$, André Kahles ${ }^{3}$, Regina Bohnert ${ }^{3}$, Géraldine Jean ${ }^{3}$, Paul Derwent ${ }^{7}$, Paul Kersey ${ }^{7}$, Eric J. Belfield ${ }^{8}$, Nicholas P. Harberd ${ }^{8}$, Eric Kemen ${ }^{9}$, Christopher Toomajian ${ }^{5}$, Paula X. Kover ${ }^{10}$, Richard M. Clark ${ }^{4}$, Gunnar Rätsch ${ }^{3}$ \& Richard Mott ${ }^{1}$

Genetic differences between Arabidopsis thaliana accessions underlie the plant's extensive phenotypic variation, and until now these have been interpreted largely in the context of the annotated reference accession Col- 0 . Here we report the sequencing, assembly and annotation of the genomes of 18 natural A. thaliana accessions, and their transcriptomes. When assessed on the basis of the reference annotation, one-third of protein-coding genes are predicted to be disrupted in at least one accession. However, re-annotation of each genome revealed that alternative gene models often restore coding potential. Gene expression in seedlings differed for nearly half of expressed genes and was frequently associated with cis variants within 5 kilobases, as were intron retention alternative splicing events. Sequence and expression variation is most pronounced in genes that respond to the biotic environment. Our data further promote evolutionary and functional studies in A. thaliana, especially the MAGIC genetic reference population descended from these accessions.

Interpreting the consequences of genetic variation has typically relied on a reference sequence, relative to which genes and variants are annotated. However, this may cause bias, because genes may be inactive in the reference but expressed in the population ${ }^{1}$, suggesting that sequencing and re-annotating individual genomes is necessary. Advances in sequencing $^{2}$ make this tractable for Arabidopsis thaliana ${ }^{3-5}$, whose natural accessions (strains) are typically homozygous. Relative to the 119-megabase $(\mathrm{Mb})$ high-quality reference sequence from Col-0 (ref. 6), diverse accessions harbour a single nucleotide polymorphism (SNP) about every 200 base pairs (bp) (ref. 3), and indel variation is pervasive $e^{3,7,8}$. Characterizing this variation is crucial for dissecting the genetic architecture of traits by quantitative trait locus mapping in recombinant inbred lines (see, for example, ref. 9) or genome-wide association in natural accessions ${ }^{10}$.

Here we have sequenced and accurately assembled the single-copy genomes of 18 accessions that, with Col- 0 , are the parents of more than 700 Multiparent Advanced Generation Inter-Cross (MAGIC) lines ${ }^{9}$, similar to the maize Nested Association Mapping (NAM) ${ }^{11}$ population and the murine Collaborative Cross ${ }^{12}$. These accessions comprise a geographically and phenotypically diverse sample across the species ${ }^{9}$. Using the genomes, seedling transcriptomes and computational gene predictions we have characterized the ancestry, polymorphism, gene content and expression profile of the accessions. We show that the functional consequences of polymorphisms are often difficult to interpret in the absence of gene re-annotation and full sequence data. The assembled genomes also contribute to the A. thaliana 1001 Genomes Project ${ }^{3-5,13}$.

\section{Genome sequencing, assembly and variants}

We assembled the 18 genomes so that single-copy loci would be contiguous, with less than one assembly error per gene, and therefore suitable for annotation. Accessions were sequenced with Illumina paired-end reads ${ }^{2}$ (Supplementary Table 1), generally with two libraries with 200-bp and 400-bp inserts and reads of 36 and $51 \mathrm{bp}$, respectively, to between 27 -fold and 60 -fold coverage. Each genome was assembled by using five cycles of iterative read mapping ${ }^{14} \mathrm{com}$ bined with de novo assembly ${ }^{15}$ (Supplementary Information sections 2 and 3, and Supplementary Tables 1 and 2). We aligned reads to the final assemblies to detect polymorphic regions ${ }^{8}$ lacking read coverage (2.1-3.7 Mb per accession; Supplementary Table 3 and Supplementary Fig. 2). At unique loci, polymorphic regions probably reflect complex polymorphisms $s^{3,8}$. The average N50 length (the contig size such that $50 \%$ of the entire assembly is contained in contigs equal to or longer than this value) of contiguous read coverage between polymorphic regions was $80.8 \mathrm{~kb}$ (Supplementary Table 4).

To report complex alleles consistently, we defined all variants against the multiple alignment consensus of Col- 0 and the assembled genomes. For each accession there were 497,668-789,187 single-base differences from Col-0, and about 45,000 ambiguous nucleotides (Supplementary Table 5). The latter may reflect heterozygosity (particularly in Po-0; Supplementary Figs 5-7) or copy-number variants, and they were largely in transposable elements and repeats covering $21.9 \%$ of the genome (Supplementary Information section 5.1, and Supplementary Figs 8 and 9). Of 3.07 million SNPs, 45.2\% were private to single accessions.

We identified 1.20 million indels, and 104,090 imbalanced substitutions, in which a sequence in Col-0 was replaced by a different sequence (Supplementary Tables 3 and 7). Although $57.5 \%$ of indels or imbalanced substitutions were shorter than $6 \mathrm{bp}, 1.9 \%$ were longer than $100 \mathrm{bp}$, and overall $14.9 \mathrm{Mb}$ of Col-0 sequence was absent in one or more accessions (Fig. 1a and Supplementary Fig. 8). The assemblies were about $1.6 \%$ and about $4.3 \%$ shorter than the reference (including

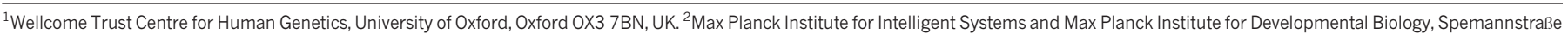

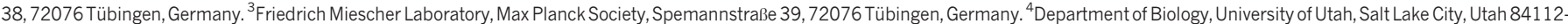
5330, USA. ${ }^{5}$ Department of Plant Pathology, Kansas State University, Manhattan, Kansas 66506-5502, USA. ${ }^{6}$ Department of Statistics, University of Oxford, South Parks Road, Oxford OX1 3TG, UK.

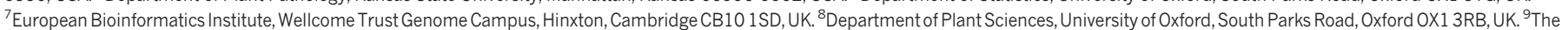
Sainsbury Laboratory, Norwich NR4 7UH, UK. ${ }^{10}$ Department of Biology and Biochemistry, University of Bath, Bath BA2 7AY, UK.

*These authors contributed equally to this work. 
a

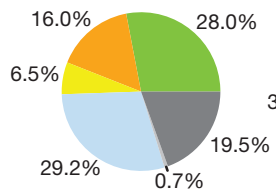

All bases (119.15 Mb)

$$
\begin{aligned}
& \text { Coding } \\
& \text { Intron }
\end{aligned}
$$
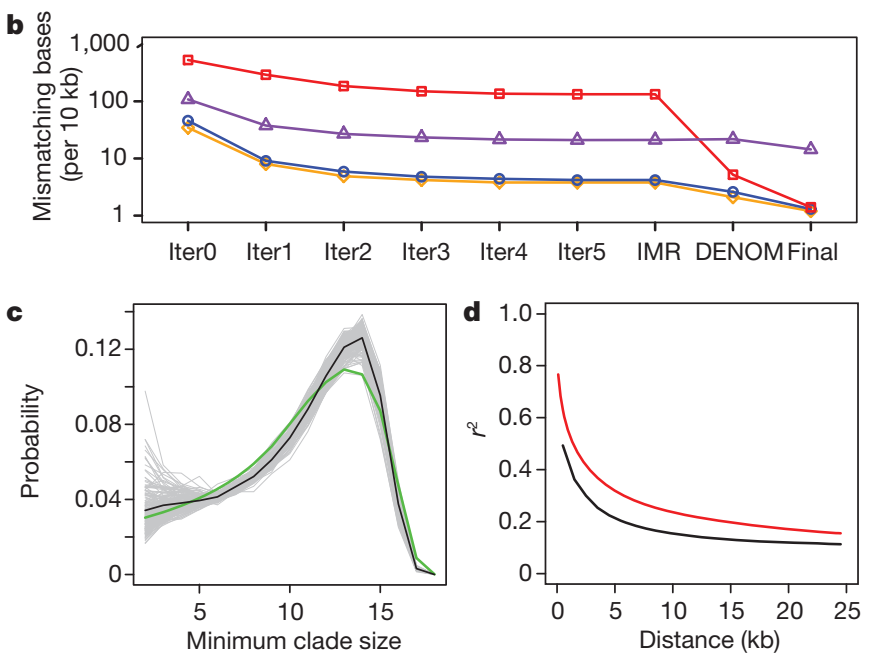

Figure 1 Assembly and variation of 18 genomes of $A$. thaliana.

a, Classification of sequence, SNPs and indels based on the Col-0 genome. b, Assembly accuracy ( $y$ axis; base substitution errors per $10 \mathrm{~kb}$ ) measured relative to four validation data sets at each of eight stages in the IMR/DENOM assembly pipeline ( $x$ axis). Bur-0 survey (blue line): 1,442 survey sequences (about 417 bp each) in predominantly genic regions ${ }^{19}$; Bur-0 divergent (red line): 188 sequences (each about 254 bp) highly divergent from Col-0 (ref. 3); Ler-0 nonrepetitive (orange line): a predominantly single-copy $175-\mathrm{kb}$ Ler-0 sequence on chromosome 5; Ler- 0 repetitive (purple line): a highly repetitive 339-kb Ler-0 locus on chromosome 3 (ref. 18; Supplementary Information section 4). Iter, iteration. c, Genome-wide distribution of the minimum clade size for all pairs of accessions (excluding Po-0). Each pair is represented by a grey line, the mean over all pairs by the black line and the random distribution by the green line. $\mathbf{d}$, Decay in linkage disequilibrium with distance (Po-0 excluded). The black line shows $r^{2}$ between SNPs; the red line shows phylogenetic $r^{2}$ (Supplementary Information section 6).

and excluding polymorphic regions, respectively), probably reflecting limitations in detecting long insertions. Although sequence differences were enriched in transposable-element and intergenic regions, about $17 \%$ of bases deleted in one or more accessions were annotated as genic in Col-0 (Fig. 1a and Supplementary Fig. 8). The density of sequence differences is greater than between classical inbred strains of mice $^{16}$, but less than between lines of maize ${ }^{17}$.

Both iterative and de novo assembly improved accuracy, with the latter being most effective at divergent loci (Fig. 1b, Supplementary Table 2 and Supplementary Fig. 10). As assessed with about $1.2 \mathrm{Mb}$ of genomic dideoxy data ${ }^{3,18,19}$ (Supplementary Information section 4), the substitution error rate was about 1 per $10 \mathrm{~kb}$ in single-copy regions, and about tenfold higher in transposable-element-rich regions. Further, RNA-seq reads covered about 100,000 SNPs per accession with $99.72 \%$ concordance (Supplementary Table 5), and junction sequences for 66 of 68 (97\%) long indels and imbalanced substitutions were confirmed by PCR and dideoxy sequencing (Supplementary Table 8). The substitution error rate for our assemblies was comparable to that reported for four other A. thaliana genome assemblies ${ }^{4}$.

\section{Genome-wide patterns of ancestry}

The ancestral relationships of the accessions vary genome-wide. We computed phylogenies ${ }^{20}$ across 1.25 million biallelic, non-private SNPs
(Supplementary Information section 6). The ancestry of each pair of accessions within a phylogeny was quantified by using the genomewide distribution of the minimum clade size of the subphylogeny containing the pair (Fig. 1c). Despite their wide geographical origins, with the exception of Po-0 and $\mathrm{Oy}-0$, all pairs have distributions similar to that of an unstructured sample. The probability of recent co-ancestry is slightly higher than expected for a few pairs of accessions, with extended haplotype sharing at a minority of loci (Supplementary Figs 11-15), perhaps reflecting selective sweeps ${ }^{7}$. Both linkage disequilibrium and correlation between neighbouring phylogenies decrease by $50 \%$ within $5 \mathrm{~kb}$ (Fig. $1 \mathrm{~d}$ and Supplementary Fig. 16). Variation among the 18 accessions is similar to a diverse global A. thaliana sample $e^{7,8}$ in nucleotide diversity (Supplementary Figs 1115), correlation with genomic features (Supplementary Tables 9-12) and structural variants (Supplementary Fig. 17).

\section{Gene annotation and transcript diversity}

A naive projection of the coordinates of the 27,206 nuclear proteincoding genes from Col-0 (TAIR10 annotation) onto the 18 genomes predicted that $93.4 \%$ of proteins were changed in at least one accession, with $32 \%$ of the total being affected by genic deletions, premature termination codons, or other disruptions (Supplementary Table 13). This large tally of disrupted genes implies that reference annotations cannot be transferred reliably; in fact, re-annotation reveals compensating changes, ensuring that many genes encode apparently functional proteins (Fig. 2a). Thus, in $96.2 \%$ of the 8,757 genes affected, the naive annotations were replaced by an alternative gene model in at least one accession (Fig. 2b and Supplementary Fig. 18). We predicted new splice sites in $64 \%$ of the 2,572 genes with splice site disruptions (in 696 cases the new sites were within 30 bp of the original ones; see, for example, Fig. 2a). Finally, there was evidence of alternative splicing in 2,106 genes (Supplementary Information sections 10.10-10.13).

For genome annotation and expression analyses (for example Figs 2-4), we generated 78-bp RNA-seq reads from two biological replicates of seedling mRNA (about 9.5 million mapped reads per accession, including Col-0; Supplementary Information section 9, and Supplementary Table 14). We integrated read alignments ${ }^{21}$ with sequence-based gene prediction $\mathrm{s}^{22}$ by using mGene.ngs (Supplementary Information sections 9-10.3, and Supplementary Fig. 19). On average, 24,681 coding genes were predicted for each accession. Comparison of Col-0 de novo predictions with TAIR10 annotations (Supplementary Table 16) showed that these predictions are more accurate (transcript $F$-score 65.2\%) than using the genome sequence $\left(\mathrm{mGene}^{22}, 59.6 \%\right)$ or RNA-seq alignments alone (Cufflinks ${ }^{23}, 37.5 \%$; Supplementary Table 17). Finally, we consolidated the de novo annotations by incorporating TAIR10 annotations where applicable (Supplementary Information section 10.4, and Supplementary Fig. 20); novel transcript structures for a known TAIR10 gene were only accepted if each newly predicted intron was confirmed by RNA-seq alignments, or if the reference gene model was severely disrupted.

We found, on average, 42,338 transcripts per accession (excluding Col-0), of which $5.5 \%(2,316)$ were novel (Table 1 and Supplementary Table 18). In each accession there were, on average, 319 novel genes (or gene fragments) supported by RNA-seq (Table 1); 717 novel genes were found in total, 496 whose sequence was present in Col- 0 but not annotated, and 221 absent from the Col-0 genome but present in the de novo assemblies of the accessions. We found protein or expressed sequence tag matches for $74.9 \%$ of the new genes, primarily from A. thaliana, A. lyrata or other Brassicaceae species (Supplementary Information sections 10.8 and 10.9).

For accession Can-0, we generated additional independent higher coverage RNA-seq data from seedling, root and floral bud, which we used to confirm $83.3 \%$ of re-annotated introns (read alignment over splice junction) and 59.9\% of transcripts (confirmation of every intron, or read coverage of $50 \%$ of the transcript for single exon 


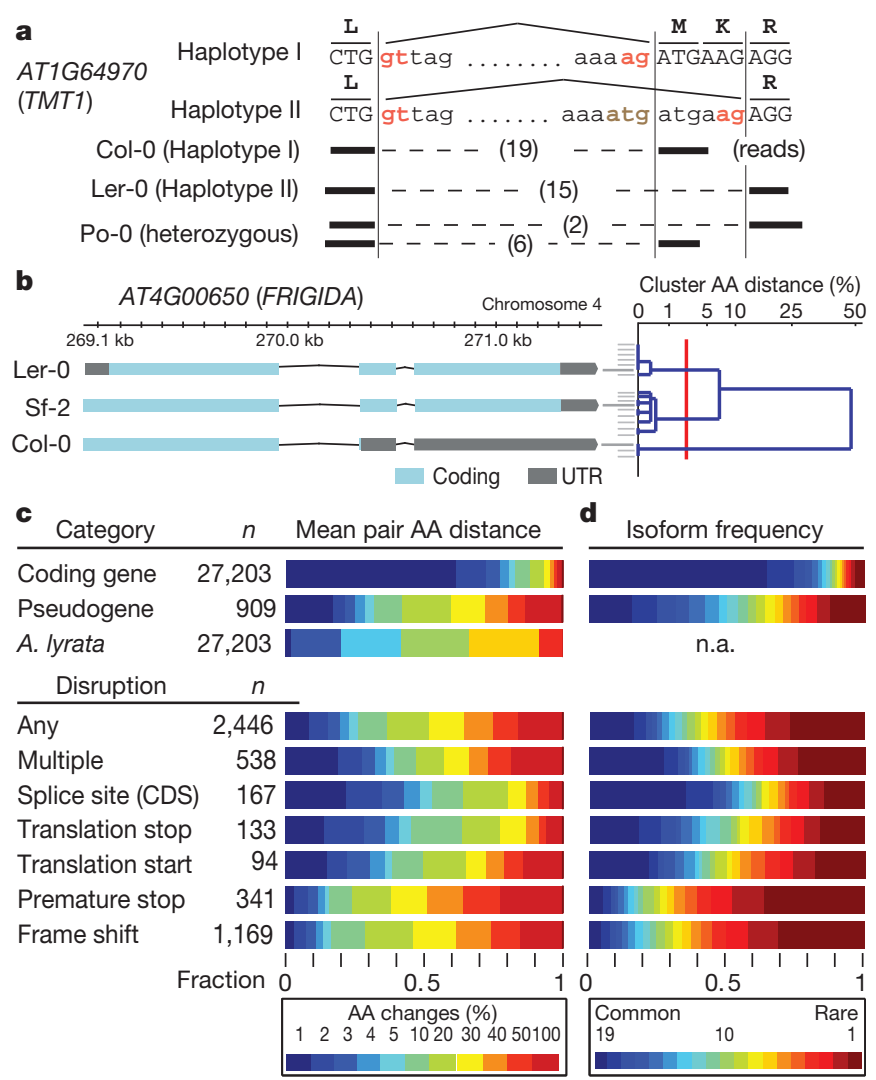

Figure $2 \mid$ Transcript and protein variation. a, Example of a splice site change between two haplotypes for the gene AT1G64970. Haplotype I (Col-0) is spliced with an intron $6 \mathrm{bp}$ (two amino acids) shorter than haplotype II (Ler-0); Po-0 (heterozygous) shows allele-specific expression of both. $\mathbf{b}$, Re-annotation of the FRIGIDA locus showing annotations for accessions Sf-2 (functional), and Col-0 (truncated by a premature stop) and Ler-0 (non-functional) (Supplementary Figs 18 and 42). Right: the 19 accessions are shown clustered on the basis of the AA distance between their FRIGIDA amino-acid sequences. Common isoform clusters (at distance $2 \%$ or less; red line) are shown, leading to three clusters with three, seven and nine accessions. c, Proteome diversity for coding genes, pseudogenes and A. lyrata genes (top) and for genes with disruptions (bottom). Reported is the fraction of genes with relative AA distance to other accessions (average over pairs) in the given colour-coded interval (Supplementary Information section 10.7). d, Frequency of isoforms of coding genes and pseudogenes (top), and those associated with different disruptions (bottom).

transcripts; Table 1). We also obtained additional RNA-seq data for Col-0 and found similar confirmation rates for the reference annotation (Supplementary Table 19). Moreover, for Can-0 we confirmed $72.1 \%$ and $84.2 \%$ of novel introns and transcripts. Many novel introns stemmed from splice disruptions that tended to be weakly expressed so RNA-seq evidence was scarcer (Supplementary Fig. 22). Finally, more than $75 \%$ of novel alternative splicing events were supported by RNA-seq (Supplementary Information section 10.5).

\section{Proteome diversity}

To understand the effect of genetic diversity on proteins, it is insufficient to study isolated DNA polymorphisms in the context of the reference annotation. We therefore defined the distance between two amino-acid (AA) sequences by the fraction of amino-acid residues that did not align identically in their global alignment. For example, for FRIGIDA, between Col-0 and Sf-2, a premature stop codon leads to an AA distance of $49 \%$ (Fig. $2 \mathrm{~b}$ ). In $77 \%$ of proteins, the mean AA distance between all accessions was less than 3\% (Fig. 2c). However, on average, 747 proteins per accession had a distance larger than $50 \%$ to any TAIR 10 protein, with markedly greater variation for pseudogenes. As expected, variation between $A$. thaliana and its congener A. lyrata ${ }^{24}$ exceeds that observed among A.thaliana accessions (Fig. $2 \mathrm{c}$ and
Supplementary Fig. 23). Disruptions to splice sites and translation start and stop codons typically caused less severe effects than premature stop codons or frame shifts (Fig. 2c) when compensating splice sites created alternative in-frame splicing (for example Fig. 2a and Supplementary Fig. 24).

Next, we identified protein isoforms across accessions (Fig. 2b, right; distinct isoforms differ by at least roughly 2\% AA distance; Supplementary Information section 10.7). For $80 \%$ of protein coding genes the most frequent isoform was very common (frequency at least 15 out of 19), whereas isoforms for pseudogenes usually occurred at lower frequency. Moreover, isoforms for large disruptions were rare (frequency 3 or less) for $37 \%$ of affected genes (Fig. 2d). This was most pronounced for premature stops and frameshifts, where purifying selection is expected to be strongest.

As expected ${ }^{3,7}$, disease resistance genes of the coiled-coil and Toll interleukin 1 receptor subfamilies of the Nucleotide-Binding Leucine Rich Repeat (NB-LRR) gene family were predicted to encode the most variable proteins (Fig. 4a and Supplementary Fig. 26). F-box and defensin-like genes implicated in diverse processes including defence ${ }^{25,26}$ were also highly variable. In contrast, housekeeping genes showed little variation.

\section{Variation in seedling gene expression}

Median expression heritability of protein-coding genes was 39\%, similar to that of novel genes (36\%) and pseudogenes (38\%), and more than for non-coding RNAs (30\%) (Supplementary Fig. 27). In total, $75 \%(20,550)$ of protein-coding genes (and $21 \%$ of non-coding RNAs and $21 \%$ of pseudogenes) were expressed in at least one accession (false discovery rate (FDR) 5\%), and 46\% (9,360) of expressed protein-coding genes were differentially expressed between at least one pair of accessions ${ }^{27}$ (Fig. 3a; FDR 5\%, Supplementary Information section 11$)$. Of these, $19 \%(1,750)$ had more than tenfold expression changes, and 1.5\% (142) more than 100-fold (Fig. 3b). For about $60 \%$ of genes, at least five accessions contributed to expression variation (Fig. 4d; Supplementary Information section 11.8).

Although the small sample size (19) precludes genome-wide association scans to identify trans expression quantitative trait loci (eQTLs), we identified potential cis-acting nucleotide variants, copy-number variants and gene structural variants (for example large indels and gene structure changes) associated with expression for 9\% (836) of differentially expressed genes (FDR 5\%; Supplementary Information section 12.2; we assessed gene-copy-number variation as in Supplementary Information section 12.4). Much of this variation was highly heritable (Fig. 3a). Consistent with identifying likely causal variants, $85 \%$ and 93\% of associated SNPs and single-nucleotide indels for cis-eQTLs were within 5 and $10 \mathrm{~kb}$ of the gene, respectively, and were strikingly concentrated in the 100-bp promoter region and $5^{\prime}$ genic sequences (Fig. 3c, d). This was also true for heritable intron retention events, in which most cis associations were within the intron or less than $1 \mathrm{~kb}$ distant (Supplementary Fig. 32). Our results corroborate the general findings $\mathrm{s}^{28-31}$ of extensive cis regulation of gene expression in A. thaliana. Neither environmental variation nor population structure markedly affected expression variation (Supplementary Information section 13). Copy-number and structural variants were associated with expression in $3 \%$ (240) of differentially expressed genes, including 45\% (64 out of 142) of genes with more than 100-fold differences (Fig. 3b), consistent with array studies ${ }^{29}$.

Differential gene expression varied by gene ontology (GO) and gene family (Fig. 4b-d, Supplementary Table 24 and Supplementary Figs 39-41). Seventeen of the 18 GO classifications that were enriched for differential expression $\left(P<10^{-3}\right)$ concerned response to the biotic environment, including pathogen defence and the production of glucosinolates ${ }^{32}$ to deter herbivores (Supplementary Table 24). These include NB-LRR genes (echoing protein variation), of which $74 \%$ were differentially expressed at up to 400 -fold change, and for which many accessions typically contributed to differential expression 

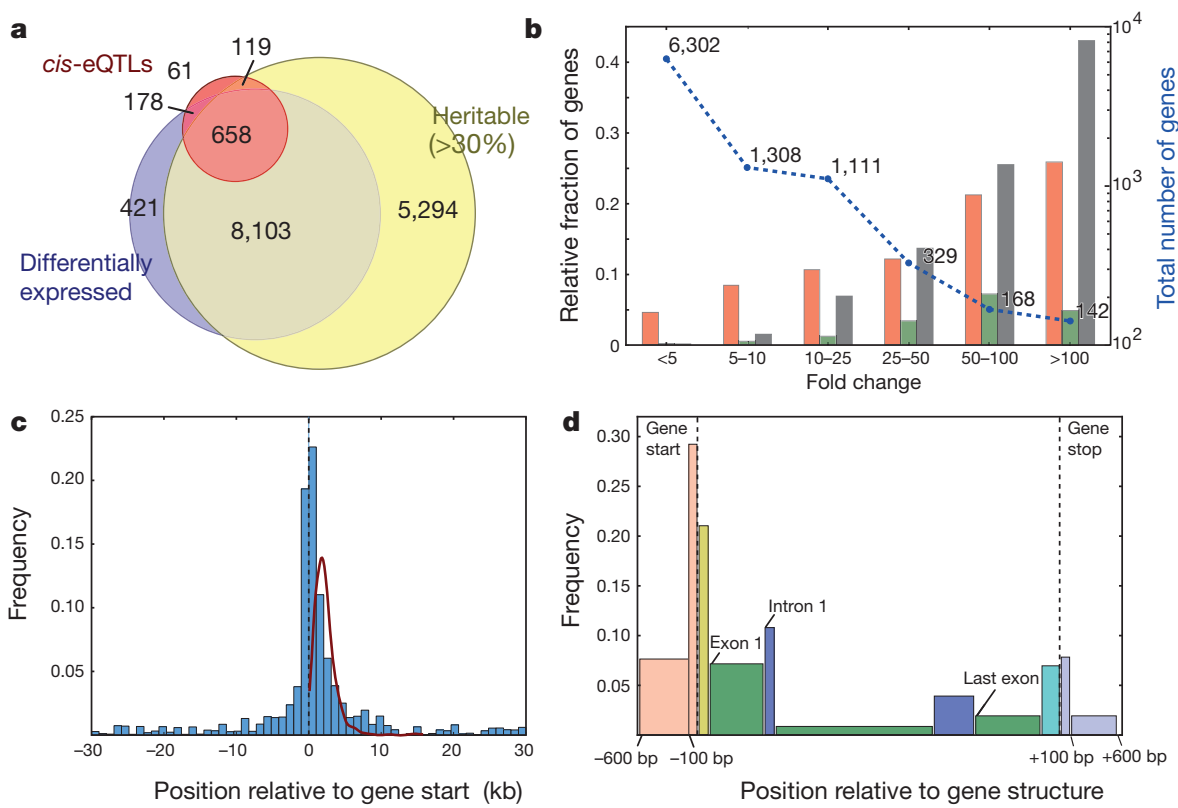

Figure $3 \mid$ Quantitative variation of coding gene expression. a, The overlap between heritable (more than $30 \%$ ) and differentially expressed (FDR 5\%) genes, and genes with a cis-eQTL (FDR 5\%). b, Differentially expressed genes and genes with ciseQTLs (FDR 5\%) categorized by fold change. Nucleotide variants (orange bars; 647 cis-eQTLs) are SNPs and single-base indels; copy-number variants (green bars; 42 cis-eQTLs) are regions with elevated coverage in aligned genomic reads in at least one accession; gene structural variants (black bars; 227 cis-eQTLs) are accession-specific deletions, insertions or changes to the gene model. c, The spatial distribution of nucleotide-variant eQTLs relative to the start of protein-coding genes (FDR 5\%, overlapping genes removed; $n=647$ ). The line shows density of gene length.

d, Frequencies of nucleotide-variant eQTLs in protein-coding genes, classified by component (bar widths are proportional to the components' average physical lengths): red bars, upstream; yellow bars, $5^{\prime}$ untranslated region; green bars, coding sequence exons; blue bars, introns; cyan bars, 3' untranslated region; grey bars, downstream.
(Fig. 4b-d). Patterns for housekeeping genes (such as ribosomal proteins, eukaryotic initiation factors or kinesins) were markedly different: although many were differentially expressed, fold changes were generally small, with variation more often being limited to a few accessions (Fig. 4b-d). Differentially expressed genes generally had much higher nucleotide diversity at synonymous sites relative to other expressed genes, a pattern also observed but less extreme at nonsynonymous sites (Supplementary Table 25). This suggests that differences in expression level were not due solely to reduced selective constraint.

The type II MADS box transcription factor family ${ }^{33}$ showed striking expression polymorphisms (Fig. 4b-d), including for the FLOWERING LOCUS $C$ (FLC $)^{34}$ and MADS AFFECTING FLOWERING (MAF) genes ${ }^{35}$. FLC, a floral inhibitor expressed highly in accessions that require prolonged cold (vernalization) to flower ${ }^{36}$, varied more than 400-fold (Supplementary Fig. 42). F-box and defensin-like genes were exceptional in that expression was restricted in a minority of genes ( $41 \%$ and $12 \%$, respectively; Fig. $4 \mathrm{~b})$, perhaps reflecting tissue-specific or environment-specific expression ${ }^{25,37}$.
Our data suggest that high turnover for some F-box families in the A. thaliana lineage ${ }^{7}$ extends to gene expression as well.

\section{Conclusion}

Our study goes beyond cataloguing polymorphisms $s^{7,17}$ to provide genome sequences for a moderately sized population sample (see also refs 4,16 ). In doing so, we were able to annotate each genome largely independently of the Col-0 reference. We found that disruptive polymorphisms were frequently compensated for, thereby conserving coding potential and highlighting the limitation of inferring consequences of polymorphisms in the absence of complete sequence data.

Our assemblies are accurate and largely complete in single-copy regions, although additional work will be needed to assemble the roughly $20 \%$ of the genome comprising repeats and transposable elements. Disentangling copy variation, long insertions and other genomic rearrangements remains a challenge. The methods we developed are of immediate relevance to the broader A.thaliana 1001 Genomes Project ${ }^{5}$ and to other organisms, and highlight the importance of RNA-seq data for annotation.

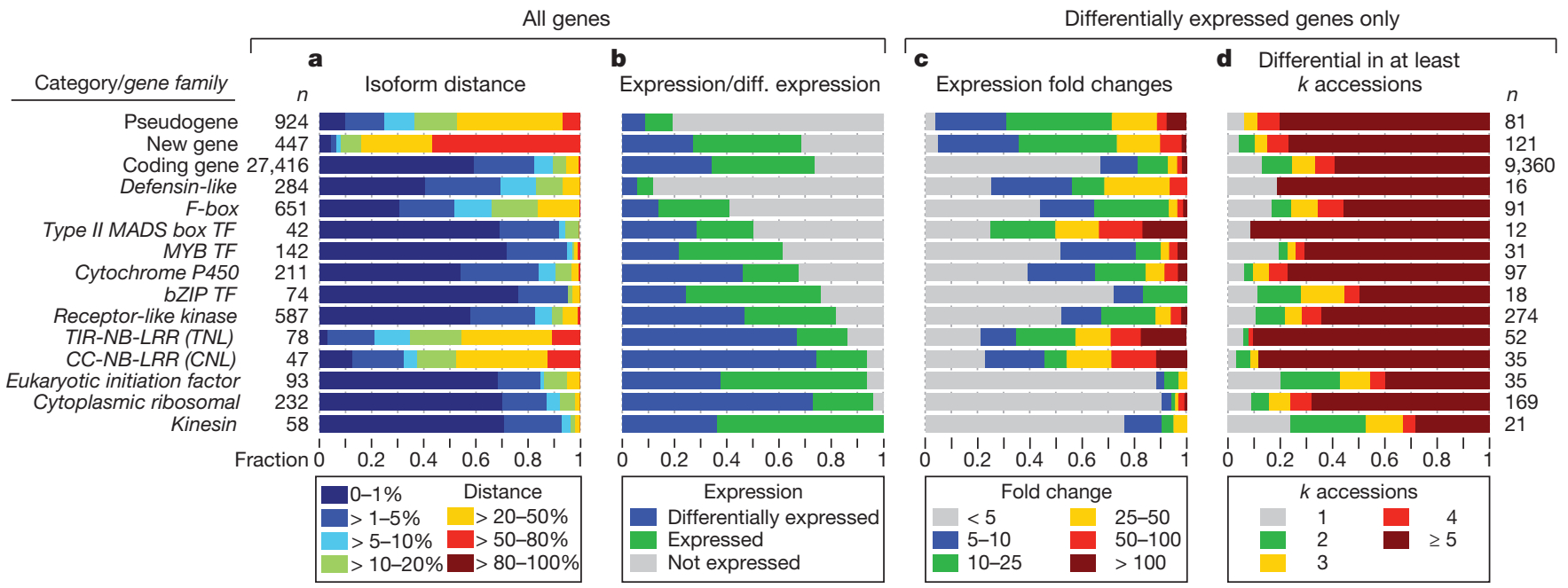

Figure $4 \mid$ Protein diversity and gene expression vary by gene category or family. The numbers next to each row are gene counts. The gene families were selected from Supplementary Figs 26 and 39-41 to represent the breadth of observed variation. a, Distribution of average AA distances to other accessions (compare with Fig. 2c). b, Fraction of unexpressed, expressed and differentially expressed genes (expressed is a superset of differentially expressed). c, Distribution of genes categorized by fold change (between lowest and highest across 19 accessions). d, Distribution of the numbers of accessions contributing to differential expression. TF, transcription factor; CC, coiled-coil; TIR, Toll interleukin-1 receptor; NB-LRR, nucleotide-binding leucine-rich repeat. 
Table 1 | Summary of gene predictions

\begin{tabular}{lrcrrr}
\hline & \multicolumn{3}{c}{ Total } & & \multicolumn{2}{c}{ Novel } \\
\cline { 2 - 3 } \cline { 5 - 6 } Type & Per accession & $\begin{array}{c}\text { RNA-seq } \\
\text { confirmed (\%) }\end{array}$ & Per accession & $\begin{array}{c}\text { RNA-seq } \\
\text { confirmed (\%) }\end{array}$ \\
\hline Genes & 33,197 & 62.7 & & 319 & 88.4 \\
Transcripts & 42,338 & 59.9 & & 2,316 & 84.2 \\
Introns & 127,640 & 83.3 & & 1,345 & 72.1 \\
Start codons & 33,264 & n.a. & & 503 & n.a. \\
Stop codons & 33,720 & n.a. & & 528 & n.a. \\
Intron retentions & 1,192 & 78.1 & & 873 & 76.5 \\
Exon skips & 80 & 80.5 & & 38 & 76.7 \\
\hline
\end{tabular}

'Total' and 'novel' are average counts over all 19 accessions. 'RNA-seq confirmed' gives the percentage fully confirmed using independent RNA-seq data (three tissues) for Can-0, the most divergent accession.

Finally, despite using only 19 accessions, we fine-mapped ciseQTLs to small genomic regions (less than $10 \mathrm{~kb}$ ), suggesting that analogous genome-wide scans in the more than 700 derived MAGIC lines could have single-gene mapping resolution for some loci. Our findings indicate that the MAGIC lines, for which population structure is largely mitigated ${ }^{9}$, will be an important and complementary resource to genome-wide association studies in A. thaliana populations ${ }^{10}$.

\section{METHODS SUMMARY}

We used the same seed stocks for Col- 0 and the 18 accessions Bur- 0 , Can- 0 , Ct- 1 , Edi-0, Hi-0, Kn-0, Ler-0, Mt-0, No-0, Po-0, Oy-0, Rsch-4, Sf-2, Tsu-0, Wil-2, Ws$0, \mathrm{Wu}-0$ and $\mathrm{Zu}-0$ that originated the MAGIC lines. DNA and RNA sequencing was performed with standard (DNA) or modified (RNA-seq) Illumina protocols. All methods are described fully in Supplementary Methods; software is available from the authors on request.

\section{Received 9 June; accepted 5 August 2011.}

Published online 28 August 2011.

1. Johanson, U. et al. Molecular analysis of FRIGIDA, a major determinant of natural variation in Arabidopsis flowering time. Science 290, 344-347 (2000).

2. Bentley, D. R. et al. Accurate whole human genome sequencing using reversible terminator chemistry. Nature 456, 53-59 (2008).

3. Ossowski, S. et al. Sequencing of natural strains of Arabidopsis thaliana with short reads. Genome Res. 18, 2024-2033 (2008).

4. Schneeberger, K. et al. Reference-guided assembly of four diverse Arabidopsis thaliana genomes. Proc. Natl Acad. Sci. USA 108, 10249-10254 (2011).

5. Weigel, D. \& Mott, R. The 1001 genomes project for Arabidopsis thaliana. Genome Biol. 10, 107, doi:10.1186/gb-2009-10-5-107 (2009).

6. The Arabidopsis Genome Initiative. Analysis of the genome sequence of the flowering plant Arabidopsis thaliana. Nature 408, 796-815 (2000).

7. Clark, R. M. et al. Common sequence polymorphisms shaping genetic diversity in Arabidopsis thaliana. Science 317, 338-342 (2007)

8. Zeller, G. et al. Detecting polymorphic regions in Arabidopsis thaliana with resequencing microarrays. Genome Res. 18, 918-929 (2008).

9. Kover, P. X. et al. A multiparent advanced generation inter-cross to fine-map quantitative traits in Arabidopsis thaliana. PLoS Genet. 5, e1000551 (2009).

10. Atwell, S. et al. Genome-wide association study of 107 phenotypes in Arabidopsis thaliana inbred lines. Nature 465, 627-631 (2010).

11. McMullen, M. D. et al. Genetic properties of the maize nested association mapping

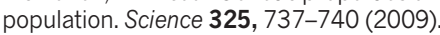

12. Durrant, $C$, et al. Collaborative cross mice and their power to map host susceptibility to Aspergillus fumigatus infection. Genome Res. 21, 1239-1248 (2011).

13. Cao, J. et al. Whole-genome sequencing of multiple Arabidopsis thaliana populations. Nature Genet. doi:10.1038/ng.911 (28 August 2011).

14. Lunter, G. \& Goodson, M. Stampy: a statistical algorithm for sensitive and fast mapping of Illumina sequence reads. Genome Res. 21, 936-939 (2011)

15. Li, R. et al. De novo assembly of human genomes with massively parallel short read sequencing. Genome Res. 20, 265-272 (2010).

16. Keane, T. M. et al. Mouse genomic variation and its effect on phenotypes and gene regulation. Nature doi:10.1038/nature10413 (in the press).

17. Gore, M. A. et al. A first-generation haplotype map of maize. Science $\mathbf{3 2 6}$, 1115-1117 (2009)

18. Lai, A. G., Denton-Giles, M., Mueller-Roeber, B., Schippers, J. H. \& Dijkwel, P. P. Positional information resolves structural variations and uncovers an evolutionarily divergent genetic locus in accessions of Arabidopsis thaliana. Genome Biol. Evol. advance online publication, doi:10.1093/gbe/evr038 (27 May 2011).

19. Nordborg, M. et al. The pattern of polymorphism in Arabidopsis thaliana. PLoS Biol. 3, e196, doi:10.1371/journal.pbio.0030196 (2005).

20. Song, Y. S. \& Hein, J. Constructing minimal ancestral recombination graphs. J. Comput. Biol. 12, 147-169 (2005).

21. Jean, G., Kahles, A., Sreedharan, V. T., De Bona, F. \& Ratsch, G. in Current Protocols in Bioinformatics Ch. 11, Unit 11.6 (Wiley, 2010).
22. Schweikert, G. et al. mGene: accurate SVM-based gene finding with an application to nematode genomes. Genome Res. 19, 2133-2143 (2009).

23. Trapnell, C. et al. Transcript assembly and quantification by RNA-Seq reveals unannotated transcripts and isoform switching during cell differentiation. Nature Biotechnol. 28, 511-515 (2010).

24. Hu, T. T. et al. The Arabidopsis lyrata genome sequence and the basis of rapid genome size change. Nature Genet. 43, 476-481 (2011).

25. Silverstein, K. A., Graham, M. A., Paape, T. D. \& VandenBosch, K. A. Genome organization of more than 300 defensin-like genes in Arabidopsis. Plant Physiol. $138,600-610(2005)$

26. Gagne, J. M., Downes, B. P., Shiu, S. H., Durski, A. M. \& Vierstra, R. D. The F-box subunit of the SCF E3 complex is encoded by a diverse superfamily of genes in Arabidopsis. Proc. Natl Acad. Sci. USA 99, 11519-11524 (2002).

27. Anders, S. \& Huber, W. Differential expression analysis for sequence count data. Genome Biol. 11, R106, doi:10.1186/gb-2010-11-10-r106 (2010).

28. Keurentjes, J. J. et al. Regulatory network construction in Arabidopsis by using genome-wide gene expression quantitative trait loci. Proc. Natl Acad. Sci. USA 104 1708-1713 (2007)

29. Plantegenet, S. et al. Comprehensive analysis of Arabidopsis expression level polymorphisms with simple inheritance. Mol. Syst. Biol. 5, 242, doi:10.1038/ msb.2008.79 (2009).

30. West, M. A. et al. Global eQTL mapping reveals the complex genetic architecture of transcript-level variation in Arabidopsis. Genetics 175, 1441-1450 (2007).

31. Zhang, X., Cal, A. J. \& Borevitz, J. O. Genetic architecture of regulatory variation in Arabidopsis thaliana. Genome Res. 21, 725-733 (2011).

32. Howe, G. A. \& Jander, G. Plant immunity to insect herbivores. Annu. Rev. Plant Biol. $59,41-66(2008)$

33. Kaufmann, K., Melzer, R. \& Theissen, G. MIKC-type MADS-domain proteins: structural modularity, protein interactions and network evolution in land plants. Gene 347, 183-198 (2005)

34. Sheldon, C. C. etal. The FLF MADS box gene: a repressor of flowering in Arabidopsis regulated by vernalization and methylation. Plant Cell 11, 445-458 (1999).

35. Ratcliffe, O. J., Kumimoto, R. W., Wong, B. J. \& Riechmann, J. L. Analysis of the Arabidopsis MADS AFFECTING FLOWERING gene family: MAF2 prevents vernalization by short periods of cold. Plant Cell 15, 1159-1169 (2003).

36. Lempe, J. et al. Diversity of flowering responses in wild Arabidopsis thaliana strains. PLoS Genet. 1, 109-118 (2005).

37. Schmid, M. et al. A gene expression map of Arabidopsis thaliana development Nature Genet. 37, 501-506 (2005).

Supplementary Information is linked to the online version of the paper at www.nature.com/nature.

Acknowledgements We thank C. Jiang and C. Brown for technical help; P. Dikjwel, K. Schneeberger and D. Weigel for providing sequence data in advance of publication; and J. Flint, M. Tsiantis, E. Feil, L. Hurst, A. Wachter and D. Weigel for comments on the manuscript. Funding was provided by the Biotechnology and Biological Sciences Research Council (BBSRC) BB/F022697/1 (to R.M. and P.X.K.), BB/D016029/2 (to P.X.K and R.M.), BB/F020759/1 (to N.P.H.) and BB/F019793/1 to P.K. (PI E. Birney), by core funding of the Max Planck Society (to G.R.), the German Research Foundation RA1894/1-1 and RA1894/2-1 (to G.R.), Volkswagen Stiftung (to O.S.), the National Science Foundation (NSF) 0929262 (to R.C. and C.T.) and 0820985 (to R.C., Principal Investigator L. Sieburth), and award no. KUK-I1-002-03 (to N.P.H), made by King Abdullah University of Science and Technology (KAUST). D. Buck, I. Ragoussis and colleagues in the Wellcome Trust Centre for Human Genetics Genomics Core performed most of the genomic sequencing, supported by the Wellcome Trust Core grant 090532/Z/09/Z. Contribution no. 11-388-J from the Kansas Agricultural Experiment Station.

Author Contributions R.M., R.C., G.R., P.X.K. and C.T. conceived and led the project. R.M. and X.G. developed and performed genome assembly and analysis. R.C, J.S. and E.O. sequenced the transcriptomes and performed analyses. R.L. performed the ancestral recombination graph analysis. C.T. and K.L.H. performed population genetics analyses. S.J.S., G.J., G.R. and A.K. developed and applied RNA-seq alignment. J.B., G.R., S.J.S., A.K. and R.B. annotated the genomes and analysed their proteins. P.D., G.R. and O.S. performed differential expression analyses. O.S. and P.D. performed genetic association analysis of expression traits. V.T.S. processed data for display in Gbrowse N.P.H., E.B. and X.G. performed experimental confirmations. P.K. and P.D. constructed the Ensembl version of the variation data. E.K. performed genomic sequencing. R.M., R.C., G.R., P.X.K., C.T., X.G. and O.S. wrote the paper with input from all authors.

Author Information DNA sequencing data are deposited in the European Nucleotide Archive (www.ebi.ac.uk/ena/) under accession number ERP000565. RNA sequencing data are deposited in the Gene Expression Omnibus (www.ncbi.nlm.nih.gov/geo/) under accession number GSE30814. Data are also available at http:// mus.well.ox.ac.uk/19genomes. Genome annotations are viewable at http:// fml.mpg.de/gbrowse-19g. Reprints and permissions information is available at www.nature.com/reprints. The authors declare no competing financial interests. Readers are welcome to comment on the online version of this article at www.nature.com/nature. This paper is distributed under the terms of the Creative Commons Attribution-Non-Commercial-Share Alike licence, and is freely available to all readers at www.nature.com/nature. Correspondence and requests for materials should be addressed to R.M. (richard.mott@well.ox.ac.uk), G.R. (Gunnar.Raetsch@tuebingen.mpg.de), R.C. (clark@biology.utah.edu), P.X.K. (p.x.kover@bath.ac.uk) or C.T. (toomajia@ksu.edu). 